\title{
Competitive Immunological Detection of Progesterone by Means of the Ion-step Induced Response of an ImmunoFET
}

\author{
R. B. M. SCHASFOORT ${ }^{a}$, b C. E. J. M. KELDERMANS ${ }^{a}$, R. P. H. KOOYMAN ${ }^{a}$, P. BERGVELD ${ }^{b}$ and J. GREVE \\ Department of Applied Physics ${ }^{a}$ and Department of Electrical Engineering ${ }^{b}$, University of Twente, \\ P.O. Box 217, 7500 AE Enschede (The Netherlands)
}

\begin{abstract}
The ion-step method was originally designed to measure immunoreactions via the change in charge density which occurs in an antibody loaded membrane, deposited on an ISFET, upon reaction with a charged antigen. In this paper we show the possibility of detecting an immunoreaction involving a non-charged antigen, progesterone, by using competitive binding of progesterone and a charged progesterone-lysozyme conjugate in the membrane. Both the immunoreaction and the non-specific binding could unequivocally be measured. The detection limit was approximately $10^{-8} \mathrm{M}$ of progesterone in the sample solution. This proves that the ion-step method is suitable for detection of both charged and non-charged antigens.
\end{abstract}

\section{Introduction}

Recently we introduced a new method to operate an immunological field-effect transistor (an ImmunoFET) by detecting a change in charge density in a membrane, deposited on an ISFET, resulting from an immunological reaction [1]. Transient potentials can be measured, arising from transient transport of ions across the membrane, which are caused by stepwise changes in electrolyte concentration (an ion-step) (see Fig. 1).

Effectively the difference in mobility of co- and counterions in the membrane is measured. These mobilities are influenced by the immobile charge density in the membrane on the ISFET, which, in turn, is determined by the proteins in the membrane. By varying the $\mathrm{pH}$, the amplitude of the transient membrane potential changes, while a certain $\mathrm{pH}$ can be found, where the amplitude of this transient changes sign. This inversion point is reached at zero effective fixed charge density and is characteristic for the type of membrane, the type of protein and the concentration of the protein in the membrane. Immunological reac- tions can be determined, provided they cause a change in charge density in the membrane. Details of the detection method are described by Schasfoort et al. $[1,2]$.

The purpose of this paper is to show an application of the ion-step method to an immunochemical model system, in which the analyte is uncharged. As a model system we have chosen the immunological reaction between progesterone and anti-progesterone. As before, the antibody is incorporated in the membrane which is deposited on an ISFET. Progesterone is an uncharged molecule, so it cannot be detected directly by using the ion-step method. However detection can

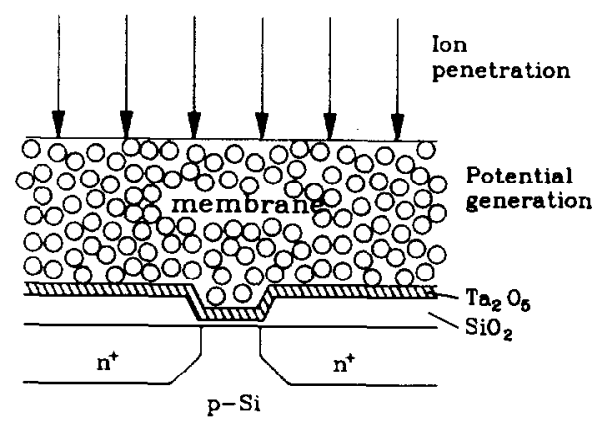

(a)

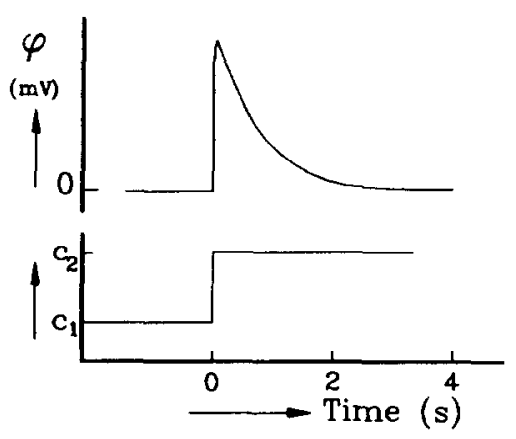

(b)

Fig. 1. (a) An ISFET exposed to an increased electrolyte concentration (an ion-step). (b) The ISFET response $\phi$ as a result of the stepwise concentration change $\left(c_{1}-c_{2}\right)$.

(C) Elsevier Sequoia/Printed in The Netherlands 
be carried out indirectly using a charge labeled progesterone conjugate, of which the concentration in the sample solution is kept constant. In this way an assay is devised based upon a competitive binding between labeled (conjugate) and non-labeled (analyte) molecules. At equilibrium the unknown progesterone concentration in the solution is then inversely related to the conjugate concentration in the membrane.

\section{Experimental}

\section{ISFET, Membrane Preparation}

The ISFETs used were n-channel, thermally grown $70 \mathrm{~nm} \mathrm{SiO}_{2}$ and $120 \mathrm{~nm} \mathrm{Ta}_{2} \mathrm{O}_{5}$ meander gates ( $15 \mu \mathrm{m}$ to $4 \mathrm{~mm}$ ), enlarged to dimensions of $3 \mathrm{~mm}$ by $5 \mathrm{~mm}$, in order to simplify the encapsulation in Hy-Sol epoxy. This encapsulation was flattened so that the ISFETs could be mounted in a leak-free flow-through system. To prepare the ISFETs for ion-step measurement, a membrane was placed on the gate of each ISFET. This support was created by depositing a 1:1 mixture of polystyrene beads (polystyrene latex $2.5 \%$, $0.12 \mu \mathrm{m}$ (Polyscience)) and agarose (0.25\% type low IEE Zero Mr. (Biorad)) on the gate. The ISFETs were then cooled to $4^{\circ} \mathrm{C}$ and the solvent was slowly evaporated, leaving a porous membrane with a thickness of approximately $4 \mu \mathrm{m}$. The ISFET was then heated to $55^{\circ} \mathrm{C}$ for $1 \mathrm{~h}$ to seal the membrane onto the gate.

\section{The Ion-step Procedure}

The procedure for determining the inversion point was standardized. The ISFET was placed in the flow-through system (see Fig. 2) between a pH electrode (Amagruss) and a reference electrode (Radiometer, Kopenhagen). A magnetic valve (General Valve Corporation, Fairfield, NJ, no. 1-29-900, 20 psi, 12 V) was used to switch between two solutions of different potassium chloride concentrations in order to obtain the transient membrane potentials at different values of the $\mathrm{pH}$. To achieve this, the buffered solutions $(10 \mathrm{mM} \mathrm{KCl}$ buffered with $0.5 \mathrm{mM}$ citric acid/KOH pH 3.8 and $10 \mathrm{mM} \mathrm{KCl}$ buffered with $0.5 \mathrm{mM}$ MES/KOH pH 6.0 (Morpholino Ethane Sulfonic acid (Merck)) are mixed in gradient vessels (see Fig. 2) and passed via the $\mathrm{pH}$ electrode to the valve, while the unbuffered high-electrolyte solution $(40 \mathrm{mM} \mathrm{KCl})$ went directly to the valve. By operating the valve, the high-electrolyte solution is allowed to pass during $1 \mathrm{~s}$ to the ISFET. Every $10 \mathrm{~s}$ a measurement was carried out at a different $\mathrm{pH}$. The time for exposing the membrane to the streaming solution was restricted to $3 \mathrm{~min}$, in order to minimize leaking out of the adsorbed proteins. This time is

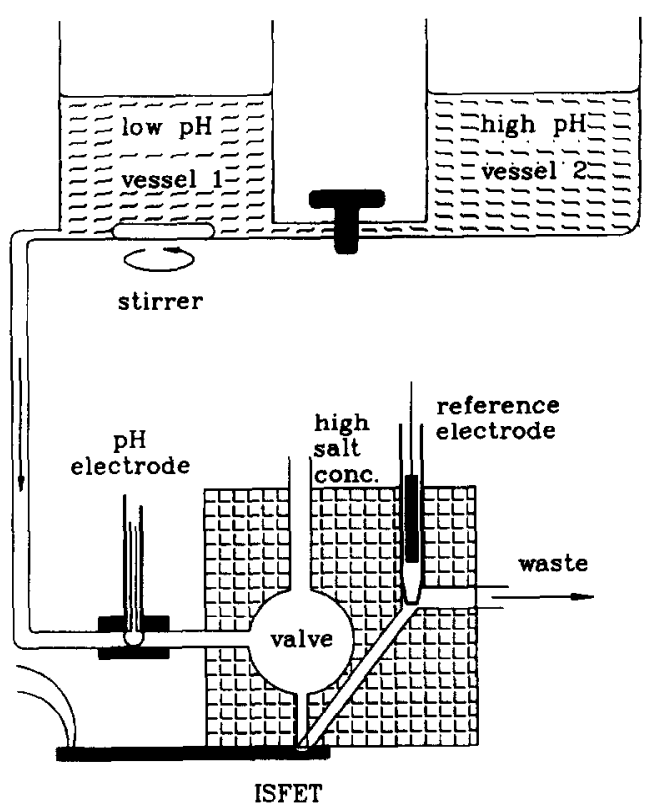

Fig. 2. Outline of the flow through system (see text).

however long enough to determine the inversion point of the transient potentials, because in $3 \mathrm{~min}$ 18 transients at different $\mathrm{pH}$ values can be recorded. The ISFET was connected to the $Y$ channel of an X-Y recorder (Kipp \& Zn, BD 90) via a source follower and a high pass filter, while the $\mathrm{pH}$ electrode was connected to the $\mathrm{X}$ channel of the recorder via a Consort $\mathrm{P} 614 \mathrm{pH} / \mathrm{mV}$ meter.

A typical registration is shown in Fig. 3, in which case the membrane contained $85 \times 10^{-6} \mathrm{M}$ of lysozyme only. Note that there is a strictly linear relationship between the membrane potential $\phi_{\mathrm{m}}$ and the $\mathrm{pH}$, with a certain inversion point $\left(\mathrm{pI}^{\prime}\right)$ and slope. The $\mathrm{pI}^{\prime}$ as well as the slope of the $\phi_{\mathrm{m}}-\mathrm{pH}$ curve change if the membrane charge density is varied by additional adsorption of

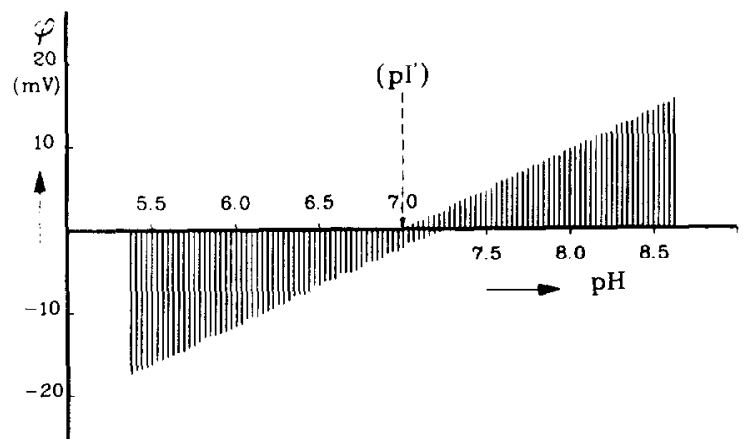

Fig. 3. Detection of the transients caused by the ion step ( $10 \mathrm{mM} \mathrm{KCl}$ to $40 \mathrm{mM} \mathrm{KCl}$ ) as a function of the $\mathrm{pH}$ of the $10 \mathrm{mM} \mathrm{KCl}$ solution using a lysozyme containing membrane. A high pass filter is used to eliminate $\mathrm{pH}$ detection. 
charged molecules. Also note that the positive and negative responses overlap near the inversion point. We observed that the width of this overlap is not always the same, probably due to inhomogeneities in the membrane. We therefore define, based on experimental reasons, the inversion point $\left(\mathrm{pl}^{\prime}\right)$ as the $\mathrm{pH}$ where the positive peak, obtained by an ion-step increase, is zero (see Fig. 3 ). The relation between membrane composition on one hand and $\mathrm{pI}^{\prime}$ and slope of the $\phi_{\mathrm{m}}-\mathrm{pH}$ curve on the other, is the key feature of the ion-step method.

\section{Monoclonal Antiserum}

Anti-progesterone (IgG) was obtained from Organon Teknika. The isoelectric point range ( $\mathrm{pI}=6.0-6.4$ ) was determined using the Phast system (Pharmacia). The cross reactivity with testosterone propionate (Sigma Chemical Co., St. Louis, MO, U.S.A.) appeared to be $<0.01 \%$, as determined with a (competition) ELISA.

\section{Synthesis of Progesterone-Lysozyme Conjugate}

Lysozyme (lysozyme hydrochloride from chicken egg white grade VI (Sigma Chemical Co., St. Louis, MO, U.S.A.)) was labeled with progesterone-1 $1 \alpha$-hemisuccinate (4-pregnen-11 $\alpha$-ol-3.20dione-hemisuccinate, Steraloids Inc., Wilton, $\mathbf{N H}$, U.S.A.) according to the mixed anhydrid method of Erlanger [3].

One milliliter $(10 \mathrm{mg} / \mathrm{ml})$ lysozyme was dialyzed overnight against distilled water to remove interfering sodium acetate. One milliliter dimethylformamide (DMF, Sigma Chemical Co., St. Louis, MO, U.S.A.) was slowly added, the solution was brought to $\mathrm{pH} 8.5$ with $1 \mathrm{M} \mathrm{NaOH}$ and cooled to $-10^{\circ} \mathrm{C}$. Then $43 \mathrm{mg}$ progesterone$11 \alpha$-hemisuccinate was dissolved in $0.5 \mathrm{ml} \mathrm{DMF}$ and cooled to $-10^{\circ} \mathrm{C}$. Fourteen $\mu$ l methylmorpholine (Merck) and $14 \mu$ l isobutylchloroformate (Merck) were added. After a $3 \mathrm{~min}$ incubation at $-10^{\circ} \mathrm{C}$, the reaction mixture was diluted with an equal volume of cold DMF; $35 \mu \mathrm{l}$ was slowly added in small portions to the lysozyme solution while keeping the temperature at $-10^{\circ} \mathrm{C}$ and the $\mathrm{pH}$ at 7-7.5 with $0.1 \mathrm{M} \mathrm{NaOH}$. The reaction was allowed to proceed at $-10^{\circ} \mathrm{C}$ for $1 \mathrm{~h}$ followed by two hours at $4{ }^{\circ} \mathrm{C}$.

The conjugate was partially purified by gelfiltration $\left(4^{\circ} \mathrm{C}\right.$, PD 10 column (Pharmacia)). The remaining unreacted progesterone derivate was removed by dialysis (Spectrapor 1, MW cut off 6000-8000, Spectrum Medical Industries Inc., LA, U.S.A.) for 3 days against $3 \times 21$ distilled water at $4{ }^{\circ} \mathrm{C}$. The conjugate was stored in small aliquots at $-20^{\circ} \mathrm{C}$. The conjugate concentration was determined with a Bradford protein assay (Biorad) with lysozyme as a standard. We found that the coupling ratio of progesterone to lysozyme was approximately $1: 1$, as determined with a competition ELISA.

\section{Results}

\section{Conjugate Preparation}

For the detection of uncharged species like progesterone it is necessary to label progesterone with a sufficient amount of charge. Then a competition experiment can be carried out, so that the unknown progesterone concentration in the solution can be correlated to the label concentration in the membrane. We tested several positively charged labels namely polylysine, polyallylamine, avidin and lysozyme. Only lysozyme appeared to be suitable as a charge label, because the polyelectrolytes polylysine and polyallylamine showed too much non-specific adsorption. Avidin (MW $60 \mathrm{kD}$ ), with its isoelectric point of about $10 \mathrm{did}$ yield a charge complex, by labeling with progesterone via a biocytin spacer (MW 372 D). However after extensive experiments we concluded that avidin resulted in a too small shift in inversion point even at maximal loading in the membrane.

A lysozyme conjugate was then prepared according to the method described in 'Experimental'. It was known from earlier experiments [2] that lysozyme, if adsorbed to a bare membrane, shifts the inversion point from $\mathrm{pI}^{\prime}=2.0$ to $\mathrm{pI}^{\prime}=7.0$. We found that the non-specific adsorption could be extensively reduced, if after adsorption of anti-progesterone at high concentration, a blocking of the remaining sites in the membrane by incubation with polyethylene glycol (MW $20 \mathrm{kD}$ ) was carried out.

\section{Immunoreaction}

The polystyrene-agarose membrane deposited on an ISFET was prepared as described in 'Experimental', followed by incubation with the monoclonal antibody anti-progesterone $(0.5 \mathrm{mg} / \mathrm{ml}$, $4{ }^{\circ} \mathrm{C}, 20 \mathrm{~h}$ ). This protein coated membrane results in an inversion point $\left(\mathrm{pI}^{\prime}\right)$ of $3.90 \pm 0.05$. Then different concentrations of the conjugate were incubated during $20 \mathrm{~h}$, in order to find the minimal concentration with maximum shift of the inversion point. We found that at $2 \times 10^{-6} \mathrm{M}$ lysozyme conjugate the maximum shift of $1.24 \mathrm{pI}^{\prime}$ was obtained. Subsequently competition experiments were carried out in duplicate with $2 \times 10^{-6} \mathrm{M}$ lysozyme conjugate and in the presence of progesterone $\left(5 \times 10^{-5} \mathrm{M}, 10^{-5} \mathrm{M}\right.$, $2 \times 10^{-6} \mathrm{M}, 4 \times 10^{-7} \mathrm{M}$ and $8 \times 10^{-8} \mathrm{M}$ ) in phosphate buffered saline. To assure that equilibrium conditions were met, incubations were carried out overnight. As a check a sample 


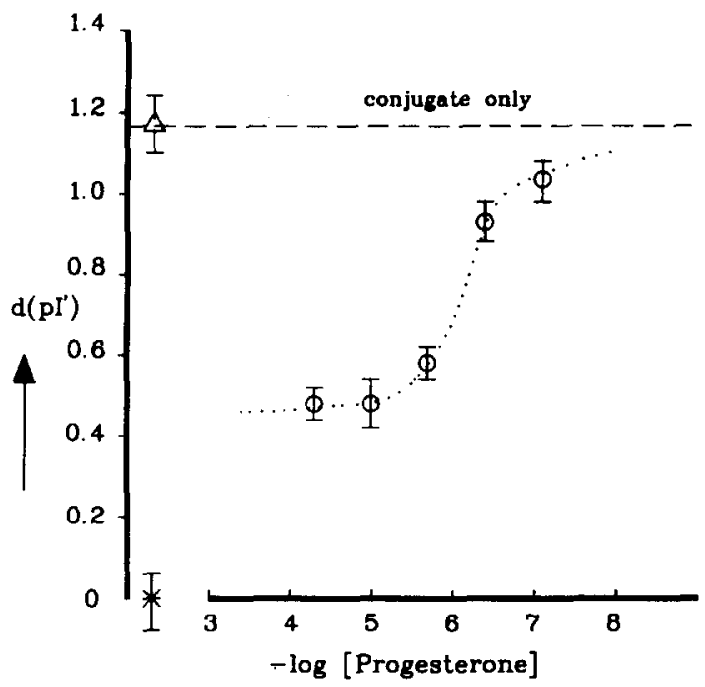

Fig. 4. Plot of the inversion point change ( $\left.\Delta \mathrm{pI}^{\prime}\right)$ as a function of the progesterone concentration $(O)$ in the sample solution. (*) Membrane coated only with anti-progesterone. The sample solution contained $2 \times 10^{-6} \mathrm{M}$ progesterone-lysozyme. Control: $10^{-4} \mathrm{M}$ testosterone propionate $(\Delta)$ in the sample solution. It is estimated that $10^{-8} \mathrm{M}$ progesterone can be detected after extrapolating the curve.

containing $100 \times 10^{-6} \mathrm{M}$ testosterone propionate and $2 \times 10^{-6} \mathrm{M}$ conjugate was measured. The results are shown in Fig. 4, where $d\left(\mathrm{pI}^{\prime}\right)=0$ is chosen as a reference point for the case that no conjugate was present. Results of independent measurements are shown in Fig. 4.

\section{Discussion}

For the competitive detection of the uncharged hormone progesterone, we could prepare a charge-label which was suited for measurements with the ion-step method. The preparation of the charge-label was not easy. Success or failure could only be tested by completion of the total procedure, since the result is also determined by the membrane properties, we used for adsorption of the antibodies. The membrane preparation was kept identical to the procedure used in earlier immuno experiments [1,2].

From the results obtained for high progesterone concentrations (see Fig. 4) we observe that a substantial part of the shift of the inversion point must be ascribed to non-specific adsorption of conjugate. Under these conditions most of the immuno reactive sites are occupied by uncharged progesterone molecules and not by the conjugate. If a low concentration of progesterone or a sample in presence of testosterone propionate is used (see right side of Fig. 4), then a shift of the inversion point as a consequence of partly the non-specific adsorption and partly the binding of conjugate to the anti-progesterone molecules in the membrane was measured. As we have used an identical conjugate concentration in the experiments, we expect that the non-specific binding results in a constant offset of the $\mathrm{pl}^{\prime}$ over the whole progesterone concentration range. The inversion point change, resulting from this nonspecific interaction of conjugate to the membrane, was $0.48 \pm 0.06 \Delta \mathrm{pI}^{\prime}$. Thus low progesterone concentrations in the sample solution result in a large shift of the inversion point. It appeared that the inversion point shift was inversely related to the progesterone concentration as expected for a competition reaction. Figure 4 seems to be a classical example of an immunological competition assay. The deviation of the duplicates was never more than $\Delta \mathrm{pI}^{\prime}=0.15$ which can be attributed to small membrane alterations. It should be kept in mind that detection was accomplished in neat sample solutions without interfering substances.

The conjugate concentration, which resulted in maximum protein coverage of the membrane after equilibration, was in agreement with earlier results of lysozyme adsorption [2]. However, if a lower conjugate concentration is used $\left(<2 \times 10^{-6} \mathrm{M}\right)$, it is expected that a progesterone concentration lower than, the presently found, $10^{-8} \mathrm{M}$ can be measured. Although the present detection limit of $10^{-8} \mathrm{M}$ is not low compared with other techniques, such as RIA and ELISA with intrinsic signal amplification, it is expected that after optimization of parameters, as membrane thickness, membrane porosity, type of conjugate, conjugate concentration, antibody coating of membrane, a lower concentration may be detectable with the ion-step method.

\section{Conclusions}

The ion-step method is unique as far as direct measurements of charge densities and isoelectric points of amphoteric macromolecules are involved. The advantage of the technique is that a direct electronic signal can be obtained as a result of an immunochemical reaction. The measurement of an uncharged hormone progesterone by using competition with a charge labeled conjugate as described in this paper, is an example of the wider applicability of the technique. We showed that the change in inversion point, after competition, is the result of a non-specific adsorption and an immunoreaction. Detection of $10^{-8} \mathrm{M}$ progesterone was possible. Higher sensitivities may be attained in the future. 


\section{Acknowledgements}

Ing. J. Bomer is acknowledged for the manufacturing of the flow-through system, Brenda Ziegler for the preliminary experiments. This investigation is part of the research program of the 'Stichting Fundamenteel Onderzoek der Materie' (FOM) which is financially supported by the 'Nederlandse Organisatie voor Wetenschappelijk Onderzoek' (NWO).

\section{References}

1 R. B. M. Schasfoort, J. Bomer, P. Bergveld, R. P. H. Kooyman and $\mathrm{J}$. Greve, Modulation of the ISFET response by an immunological reaction, Sensors and Actuators, 17 (1989) $531-535$.

2 R. B. M. Schasfoort, R. P. H. Kooyman, P. Bergveld and J. Greve, A new approach to ImmunoFET operation, Biosensors, accepted for publication.

3 B. F. Erlanger, Steroid-protein conjugates, J. Biol. Chem., 228 (1957) 713. 